\title{
Milliliter per Square Meter per Hour
}

National Cancer Institute

\section{Source}

National Cancer Institute. Milliliter per Square Meter per Hour. NCI Thesaurus. Code C73762.

A dose calculation unit expressed in milliliter(s) per square meter per period of time equal to sixty minutes. 